Check for updates

Cite this: RSC Adv., 2018, 8, 8920

Received 25th December 2017 Accepted 8th February 2018

DOI: $10.1039 / c 7 r a 13634 j$

rsc.li/rsc-advances

\section{Effect of multi-walled carbon nanotubes on the physical properties and crystallisation of recycled PET/TPU composites}

\author{
Changqing Fang, (D) *ab Rong Yang, ${ }^{a}$ Zisen Zhang, ${ }^{a}$ Xing Zhou, ${ }^{a}$ Wanqing Lei, ${ }^{\text {ab }}$ \\ Youliang Cheng, ${ }^{a}$ Wei Zhang ${ }^{a}$ and Dong Wang ${ }^{a b}$
}

Thermoplastic polyurethane (TPU) was blended with recycled polyethylene terephthalate (rPET) to prepare rPET/thermoplastic polyurethane (TPU) composites. Meanwhile, multiple-walled carbon nanotubes (MWCNTs) were employed as a reinforcing filler to study the synergistic effect between CNTs and rPET/ TPU composites. The effect of CNT content on the morphology and micro-structure of the composites was investigated using a scanning electron microscope (SEM) and X-ray diffraction (XRD). The thermal properties were characterized using differential scanning calorimetry (DSC), thermogravimetric analysis (TGA) and dynamic mechanical analysis (DMA). The mechanical properties were investigated using tensile tests and hardness measurements. The results showed that TPU was compatible with rPET. The existence of rPET changed the crystalline phase and affected the glass transition and crystallisation temperature of the TPU matrix. The rPET/TPU composites displayed poor thermal stability and tensile properties when compared to pure TPU. The addition of CNTs had no effect on the crystalline phase of the rPET/TPU composites. Due to the occurrence of interfacial adhesion between the CNTs and rPET, the CNTs displayed an offset effect on the reaction of rPET and decreased the rigidity of the molecular chain in the rPET/TPU composites. The thermal stability and tensile strength of the CNTs/rPET/TPU composites improved with an increase in CNT content.

\section{Introduction}

As is known, poly(ethylene terephthalate) (PET) is a typical semicrystalline thermoplastic polymer with good thermal and mechanical properties. It is used extensively in disposable beverage bottles and food packaging owing to its nontoxic nature, durability and crystal clear transparency. ${ }^{\mathbf{1 - 4}}$ With the considerable consumption of food and beverages, flexible manufacturing, printed electronics, etc., PET waste has increased drastically. ${ }^{5}$ The use of PET not only brings about serious environmental problems, but also causes a shortage of energy as it is not a biodegradable material. Hence, recycling of waste PET products has become an increasingly hot issue around the world.

At present, there are two commonly employed methods for recycling PET waste: physical recycling and chemical recycling. Physical recycling methods, i.e. polymer blending, provide a new way to recycle waste, offering an opportunity to use PET as a modifying agent in blended polymer materials for commercial applications. ${ }^{6}$ In previous studies, the blending of polymer

${ }^{a}$ Faculty of Printing, Packing Engineering and Digital Media Technology, Xi'an University of Technology, Xi'an 710048, P. R. China. E-mail: fcqxaut@163.com

${ }^{b}$ Faculty of Mechanical and Precision Instrument Engineering, Xi'an University of Technology, Xi'an 710048, P. R. China materials with recycled PET has been investigated. Many works have been devoted to exploring polymer-polymer microfibrillar reinforced composites, such as $\mathrm{PET} /$ high density polyethylene (HDPE), ${ }^{6}$ PET/low density polyethylene (LDPE), ${ }^{7,8}$ PET/isotactic polypropylene (iPP) ${ }^{\mathbf{9}, 10}$ and PET/concrete composites., ${ }^{\mathbf{4 1 1}}$ PET not only increases the tensile properties of polymers but also improves the dead load and acid resistance of concrete building materials.

Thermoplastic polyurethane (TPU) is a kind of polymer material that has excellent physical properties and biocompatibility. It is widely used in daily life, and in the fields of industry, agriculture and medicine, such as in roller systems, tread, automotives, wire and cable and biomedical products. ${ }^{12}$ It is known that the elastomer polymer materials can be reinforced via blending with a rigid thermoplastic material. For example, V. Jašo et al. ${ }^{13}$ prepared TPU/polylactic acid (PLA) composites, and found that the composites had an improved tensile strength and retained relatively high elongation. A rigid thermoplastic material PET has also been used to blend with TPU elastomers in previous work; ${ }^{14}$ Manfred et al. ${ }^{14}$ set the heat temperature of an extruder to $220^{\circ} \mathrm{C}$, which was below the melt temperature of the PET material. Hence, TPU and PET were proved to be incompatible. Polymer matrix-added carbon nanotubes (CNTs) have received considerable attention over nearly two decades as some of the properties of polymer- 
polymer microfibrillar reinforced composites are not yet perfect. Due to their high strength and high elastic modulus along with their large aspect ratio, CNTs have been proved to be an effective reinforcing element in CNTs/polymer composites. ${ }^{\mathbf{1 1 2 , 1 5 - 1 7}}$ The nucleation effect occurs between CNTs and semi-crystalline polymers since CNTs have a similar chain-like structure to polymer molecular chains, which can accelerate the crystallization kinetics of polymer matrices. The properties of CNT-based polymer composites greatly depend on the interfacial interaction between the CNTs and the polymer matrix. ${ }^{18,19}$ For example, Chen et al. ${ }^{18}$ investigated the effect of CNT content on the strain sensitivity and shape memory behavior of CNTs/PU composites. Steinert et al. ${ }^{20}$ studied the electrical properties of PET/carbon nanotube composite films, and found that the electrical conductivity was very susceptible to the content and dispersion of CNTs. The electrical properties of the PET/CNTs nanocomposites containing only 1 wt $\%$ MWCNTs were nine orders of magnitude higher than those of pure PET. ${ }^{21}$ CNTs are also used to modify the properties of polymer-polymer composites. ${ }^{2-24}$ For example, when the content of MWCNTs was $5 \mathrm{wt} \%$ in PET/LDPE blends (mass ratio $=20: 80$ ), Cardinaud et al. ${ }^{22}$ found that the MWCNTs had little effect on the rheological properties of the blends. The melt viscosity increased as the content of the MWCNTs increased. Most researchers have only proven the effect of the interfacial interactions of CNTs on the properties of a single polymer such as PET and TPU or other polymer-polymer composites. However, little research has focused on exploring the effect of CNTs on the properties of PET/TPU composites, especially for waste PET.

Hence, in this work, we prepared rPET/TPU composites with the aim of recycling waste PET and introducing different amounts of CNTs into the composite to further analyze the relationship between the CNT content and the properties of the CNTs/rPET/TPU composites. To the best of our knowledge, this is the first study on the effect of CNTs on the properties of rPET/ TPU composites, and it was found that rPET and TPU have good compatibility and the CNTs have an offset effect on the rPET/ TPU composites. The composite products can be used in packing bags for express service packages. It is meaningful to protect the environment and turn waste into treasure.

\section{Experimental}

\subsection{Materials}

Waste PET mineral water bottles were collected and utilized. After removing the caps and labels, the bottles were cut into flakes (average size $5 \mathrm{~mm} \times 5 \mathrm{~mm}$ ) which were washed with alcohol, hydrochloric acid and deionized water to remove adhesive materials and dust, and then dried at room temperature. The melt flow index of the waste PET flakes was $15.12 \mathrm{~g} \mathrm{~s}^{-1}$ (at a load of $0.35 \mathrm{~g}$ ). Multi-walled carbon nanotubes (MWCNTs) were purchased from Beijing BOYU GAOKE New Materials Co., Ltd. China. Their diameter was 20-40 $\mathrm{nm}$ and their length was about 10-30 $\mu \mathrm{m}$. Hydrochloric acid ( $\mathrm{HCl}, 37 \mathrm{wt} \%$ ) was purchased from Sichuan XILONG Chemical Reagent Co., Ltd. China. Deionized water was used in the process of cleaning the PET sheets. TPU (tradename of Elastollan), with a density of $1.14 \mathrm{~g} \mathrm{~cm}^{-3}$ and a vicat softening point temperature of about $116{ }^{\circ} \mathrm{C}$, was purchased from Dongguan city Zhangmutou embellish plastic raw materials sales department.

\subsection{Sample preparation}

Before the blending process, the CNTs were treated with concentrated nitric acid at $60{ }^{\circ} \mathrm{C}$ for $10 \mathrm{~h}$ and then dried in a drying oven for $3 \mathrm{~h}$ at $400{ }^{\circ} \mathrm{C}$ for the purpose of obtaining purified CNTs. ${ }^{25}$ The TPU pellets and PET flakes were dried at $80^{\circ} \mathrm{C}$ for $10 \mathrm{~h}$. The mass ratio of rPET and TPU was $1: 10$. The prepared mixture was placed in a double-screw extruder $(\mathrm{SHJ}-$ 35 , Nanjing, China) at a set temperature profile of $260{ }^{\circ} \mathrm{C}$, $260{ }^{\circ} \mathrm{C}, 245^{\circ} \mathrm{C}, 230{ }^{\circ} \mathrm{C}, 220^{\circ} \mathrm{C}, 200{ }^{\circ} \mathrm{C}$. The feed speed was set at $10 \mathrm{rpm}$. In this work, the mass ratio of rPET and TPU was $1: 10$. The CNT content was varied from $0.3 \mathrm{wt} \%$ to $2 \mathrm{wt} \%$ relative to the mass of rPET. For the sake of comparison, pure TPU and $\mathrm{rPET} / \mathrm{TPU}$ (mass ratio $=1: 10$ ) were also processed under the same conditions. The abbreviations of the composites synthesized from rPET, TPU and CNTs (0.3 wt\%, $0.5 \mathrm{wt} \%$, $0.75 \mathrm{wt} \%, 1 \mathrm{wt} \%$ and $2 \mathrm{wt} \%$ relative to the mass of $\mathrm{rPET}$ ) are defined as rPET/TPU1C, rPET/TPU2C, rPET/TPU3C, rPET/ TPU4C and rPET/TPU5C, respectively (as shown in Table 1). The composites were then compression-molded in a hot press machine (JXF, Shanghai, China). Firstly, the samples were heated for $10 \mathrm{~min}$ at a melt temperature of $200{ }^{\circ} \mathrm{C}$ and compressed at a pressure of $19 \mathrm{MPa}$. Then, they were compressed at $23 \mathrm{MPa}$ for $5 \mathrm{~min}$ and deflated for $2 \mathrm{~min}$, and this was performed twice. Finally, the samples were repressed at $23 \mathrm{MPa}$ for $5 \mathrm{~min}$ to obtain sheets with a thickness of $1.6 \mathrm{~mm}$.

\subsection{Morphology}

The morphology of the samples was observed using a scanning electron microscope (SEM) JSM-6700M with an accelerating voltage of $1 \mathrm{kV}$. The samples were fractured in liquid nitrogen and the fractured surfaces were observed.

Table 1 Recipe for the preparation of CNTs/rPET/TPU composites (g)

\begin{tabular}{lccccc}
\hline Sample & rPET/TPU1C & rPET/TPU2C & rPET/TPU3C & rPET/TPU4C & rPET/TPU5C \\
\hline CNTs & 0.5 & 0.5 & 0.5 & 0.5 & 0.5 \\
PET & 166.67 & 100 & 66.67 & 50 & 25 \\
TPU & 1666.7 & 1000 & 666.7 & 500 & 250 \\
CNTs/PET (wt\%) & 0.3 & 0.5 & 0.75 & 2
\end{tabular}


XRD analysis of the samples was performed using an XRD-7000 (SHIMADZU LIMITED, Japan). All of the samples were scanned at $2 \theta$ angles from $10-70^{\circ}$ and a scanning speed of $8.00 \mathrm{deg} \mathrm{min}^{-1}$.

\subsection{Thermal properties}

Differential scanning calorimetry (DSC) analysis was carried out using NETZSCH DSC200F3 equipment. The masses of the samples were approximately 5-10 $\mathrm{mg}$. Firstly, the measurements were conducted at a heating rate of $10{ }^{\circ} \mathrm{C} \mathrm{min}^{-1}$ from $-80{ }^{\circ} \mathrm{C}$ to $250{ }^{\circ} \mathrm{C}$, and then maintained for $1 \mathrm{~min}$ at $250{ }^{\circ} \mathrm{C}$ in order to eliminate the thermal history, the system was then cooled to $-80{ }^{\circ} \mathrm{C}$ at $10^{\circ} \mathrm{C} \min ^{-1}$, and finally reheated to $250{ }^{\circ} \mathrm{C}$ at $10{ }^{\circ} \mathrm{C} \min ^{-1}$ under a nitrogen flow to obtain the glass transition temperature $\left(T_{\mathrm{g}}\right)$, crystallisation temperature $\left(T_{\mathrm{c}}\right)$, melt temperature $\left(T_{\mathrm{m}}\right)$, crystallisation enthalpy $\left(\Delta H_{\mathrm{c}}\right)$ and melting enthalpy $\left(\Delta H_{\mathrm{m}}\right)$.

The thermal stability of the samples was measured using thermogravimetric analysis (TGA) with a NETZSCH TG209F3. The weight of the samples was 5-10 mg. Tests were conducted

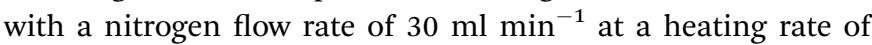
$10{ }^{\circ} \mathrm{C} \mathrm{min}{ }^{-1}$ from 30 to $600{ }^{\circ} \mathrm{C}$.

\subsection{Dynamic mechanical analysis (DMA)}

Dynamic mechanical analysis (DMA) was performed using a METTLER TOLEDO DMA 1 STARe system. Tests were conducted in the tensile module, at $1 \mathrm{~Hz}$ and a heating rate of $3{ }^{\circ} \mathrm{C} \mathrm{min}{ }^{-1}$ from -100 to $150{ }^{\circ} \mathrm{C}$. A rectangular strip (dimensions $20 \mathrm{~mm} \times 10 \mathrm{~mm} \times 1.6 \mathrm{~mm}$ ) was cut from the hot pressed sheet.

\subsection{Mechanical properties}

The tensile properties of the samples were investigated using a universal tensile machine at a cross head speed of 50 $\mathrm{mm} \min ^{-1}$ and at room temperature. Tests were performed using a dumb-bell specimen (according to ISO527-2/1BA/50). The gauge length and thickness of the specimen were $31.7 \mathrm{~mm}$ and $1.6 \mathrm{~mm}$, respectively.

\subsection{Hardness testing}

The hardness testing of the composite samples was conducted using a TH200 Shore A durometer, according to GB/T531-92. Samples were cut from the hot pressed sheets. At least five points were tested for each sample to obtain an average, and the distance between two points was $6 \mathrm{~mm}$.

\subsection{Fourier transform infrared spectrometry (FT-IR)}

FT-IR was performed on a film on $\mathrm{KBr}$ formed tablets to characterize the purified CNTs, and the FT-IR spectra were recorded as a film on $\mathrm{KBr}$ formed tablets under a vacuum press in the spectrophotometer (SHIMADIU FTIR-8400S (CE)), in the wave band ranging from 400 to $4000 \mathrm{~cm}^{-1}$. An average of 128 scans were run to reach a resolution of $4.0 \mathrm{~cm}^{-1}$.

\subsection{Raman spectra}

Raman testing was performed on a LabRAM HR 800 (HORIBA JOBIN YVON) using a $633 \mathrm{~nm}$ excitation line from a HeNe laser with a power of about $0.5 \mathrm{~mW}$. The wavenumber range was 100$3500 \mathrm{~cm}^{-1}$.

\section{Results and discussion}

\subsection{Morphological characterization}

Micrographs of the cryogenically fractured surfaces of pure TPU and CNTs/rPET/TPU composites are shown in Fig. 1. Pure TPU showed typical block copolymer morphology, with the spheres representing the hard segment phase and the base representing the soft segment phase. The fractured surface of pure rPET was flat and pure rPET displayed a homogeneous continuous phase. As shown in Fig. 1(b-g), all of the micrographs displayed a single-phase structure, suggesting that TPU was compatible with rPET in this work. It is easy to observe that there are obvious differences in the distribution of the soft and hard segments. The existence of rPET changed the typical morphology of TPU.

In order to characterize the surface of the purified CNTs, FT-IR and Raman testing were also adopted. As shown in Fig. 2a, the FTIR spectrum of the purified CNTs showed characteristic peaks corresponding to the stretching of $\mathrm{C}-\mathrm{C}$ at $1573 \mathrm{~cm}^{-1}$, the $\mathrm{C}=\mathrm{O}$ stretching in $-\mathrm{COOH}$ at $1735 \mathrm{~cm}^{-1}$ along with $-\mathrm{OH}$ at $3649 \mathrm{~cm}^{-1}$, suggesting that the surface of the CNTs is made up of hydroxyl groups and carboxyl groups. Fig. 2b shows the Raman spectrum of the purified CNTs, and the peaks at $1327 \mathrm{~cm}^{-1}, 1579 \mathrm{~cm}^{-1}$ and $2656 \mathrm{~cm}^{-1}$ are assigned to the $\mathrm{D}$ mode, $\mathrm{G}$ mode and $\mathrm{G}^{\prime}$ mode, respectively. The D mode represents lattice defects. The ratio of $I_{\mathrm{D}} /$ $I_{\mathrm{G}}$ is $c a$. 1.16, which indicates the degree of functionalization of the purified CNTs. After introducing purified CNTs into the rPET/ TPU composites, short CNT microfibers exposed on the matrix surface can be observed [Fig. 1c and d]. With increasing CNT content, the distribution of short microfibers in the samples became more dense. In composites with 0.75 to 2 wt\% CNTs [Fig. 1e-g], there were no short microfibers and the CNTs were wrapped in the composites, revealing the stronger interfacial adhesion between the CNTs and the rPET/TPU composites. This is because after being acidified, the surface of the CNTs was made up of hydroxyl groups, carboxyl groups and other groups, which increases the dispersion of the carbon nanotubes in the rPET/TPU composites. Also, since CNTs have a similar chain-like structure to polymer molecular chains, the nucleation effect occurs between the CNTs and the composite, which can contribute to interfacial adhesion in the matrix. The composites with 1 and $2 \mathrm{wt} \%$ CNTs had a homogeneous phase morphology. A more homogeneous phase morphology appeared in the composite with 2 wt\% CNTs and most of the CNTs were in contact with each other, showing obvious interfacial adhesion between the CNTs and the matrix. In all of the samples, good bonding between the CNT microfiber phase and the matrix was observed, moreover, no serious aggregation morphology appeared and the CNTs exhibited a homogeneous dispersion in the matrix.

X-ray diffraction analysis was carried out to investigate the development of the crystalline phase in all of the samples. TPU is a typical segmented copolymer that consists of alternating soft polyester and hard polyurethane-urea segments. At a certain temperature, these segments show micro-phase separation, 

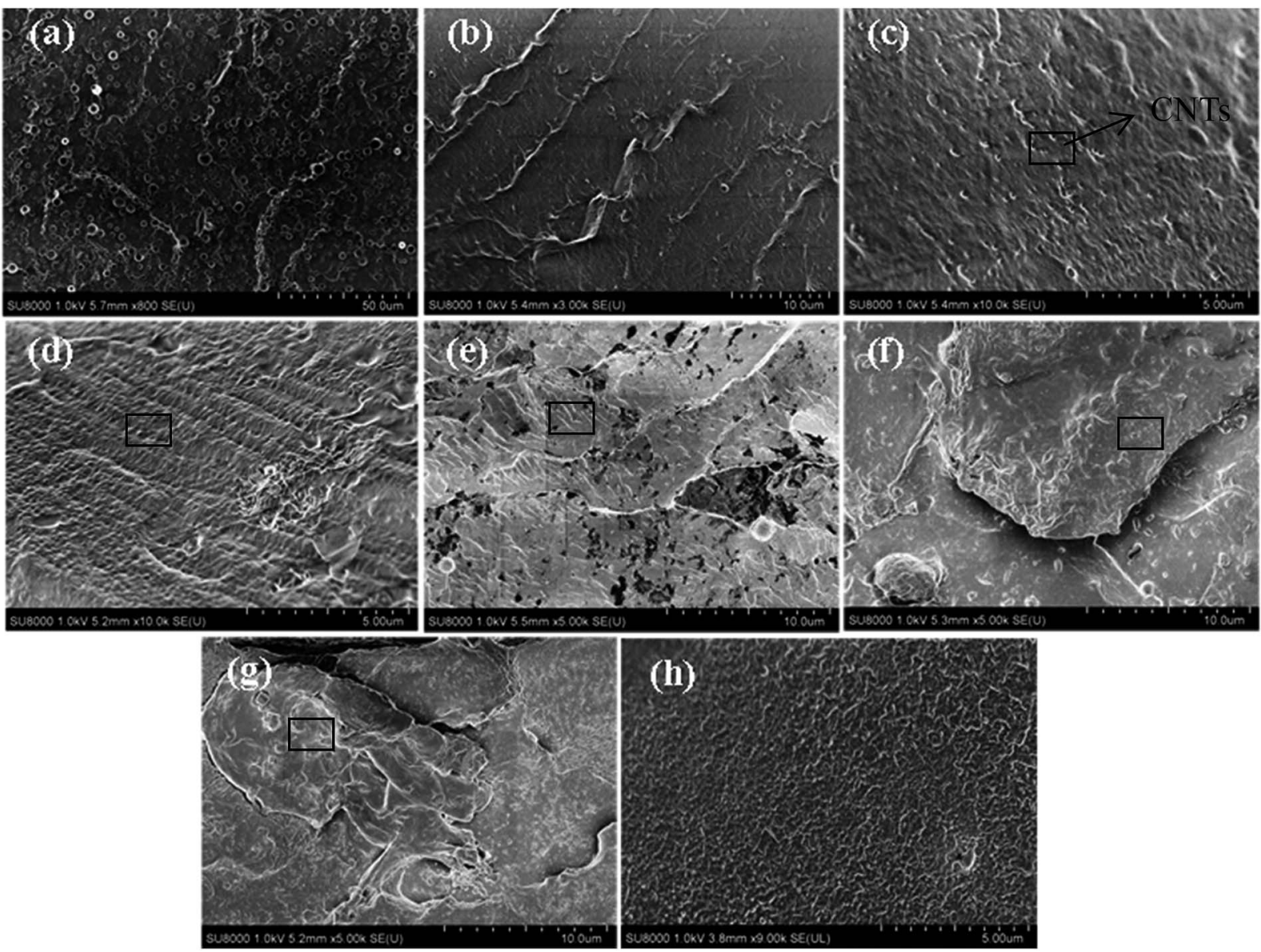

Fig. 1 Scanning electron microscope (SEM) microimages of pure TPU and CNTs/rPET/TPU composites. The cryogenically fractured surfaces of the samples were coated with gold. (a) Pure TPU, (b) rPET/TPU, (c) rPET/TPU1C, (d) rPET/TPU2C, (e) rPET/TPU3C, (f) rPET/TPU4C, (g) rPET/ TPU5C and (h) pure rPET.

forming hard and soft phases. ${ }^{17}$ As shown in Fig. 3, one characteristic diffraction peak of pure TPU appeared at $2 \theta=21.52^{\circ}$, which corresponds to the soft segments of segmented PU. ${ }^{26}$ Pure rPET displayed two diffraction peaks, at $22.42^{\circ}$ and $25.82^{\circ}$. The characteristic diffraction peak of rPET/TPU composites was at $2 \theta=19.38^{\circ}$, and the change in the position of the soft segment in segmented PU was attributed to the introduction of rPET, which contains ester groups (-COOR) crystalline regions, ${ }^{27}$ suggesting (a)

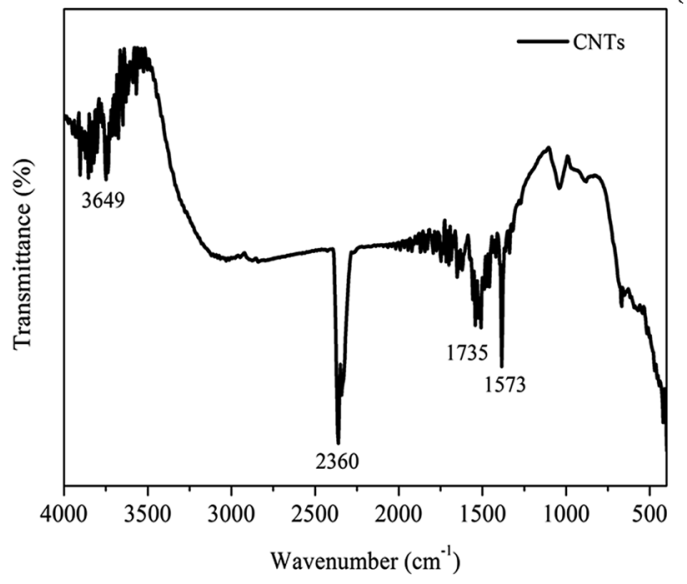

(b)

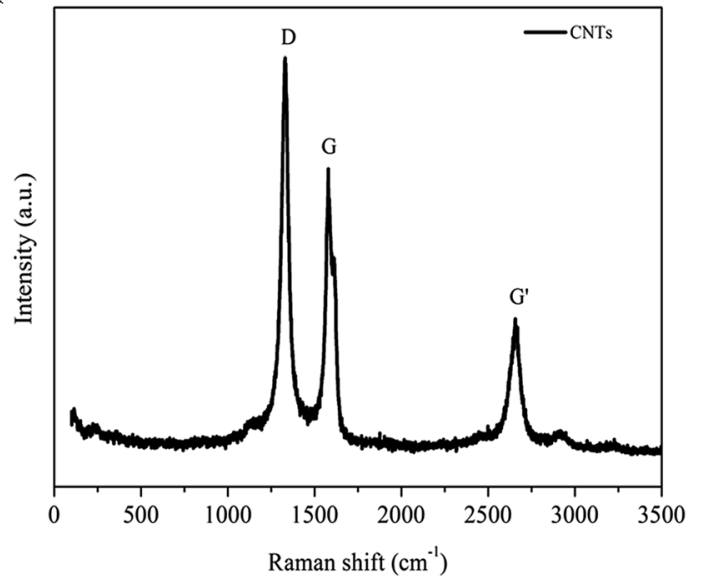

Fig. 2 (a) FT-IR and (b) Raman spectra of purified CNTs. 


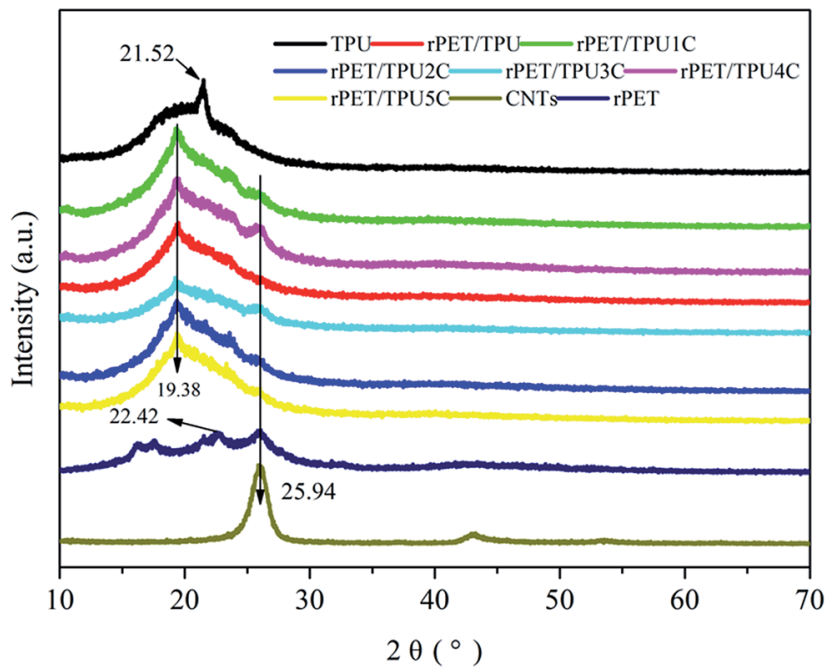

Fig. 3 XRD patterns of pure rPET, pure TPU, purified CNTs and rPET/ TPU and CNTs/rPET/TPU composites.

that the existence of rPET can change the crystalline phase of the TPU matrix. For the CNTs/rPET/TPU composites, it can be clearly observed that there are two diffraction peaks at $2 \theta=19.38^{\circ}$ and $25.94^{\circ}$. The peak that appears at $25.94^{\circ}$ was related to the multiwall interlayer spacing of the nanotubes $\mathrm{d} 002,{ }^{28,29}$ as observed in the curve of the CNTs sample. There were no changes in the position and relative intensity of the $\mathrm{rPET} / \mathrm{TPU}$ composite diffraction peak throughout the whole range of the CNTs/rPET/ TPU composites, indicating that the addition of CNTs does not change the crystalline phase of rPET/TPU composites.

\subsection{Thermal characterization}

The compatibility of several component polymers can be investigated using DSC analysis. The thermal properties can offer insight into the interaction between components in the composites. Table 2 shows the glass transition $\left(T_{\mathrm{g}}\right)$, crystallisation $\left(T_{\mathrm{c}}\right)$ and melt $\left(T_{\mathrm{m}}\right)$ temperature, and the crystallisation $\left(\Delta H_{\mathrm{c}}\right)$ and melting enthalpy values $\left(\Delta H_{\mathrm{m}}\right)$ of pure rPET, pure TPU and rPET/TPU and CNTs/rPET/TPU composites. DSC curves are shown in Fig. 4. Pure TPU has a glass transition at around $-36.2{ }^{\circ} \mathrm{C}$ and rPET at $-15.2{ }^{\circ} \mathrm{C}$. Pure TPU melted at

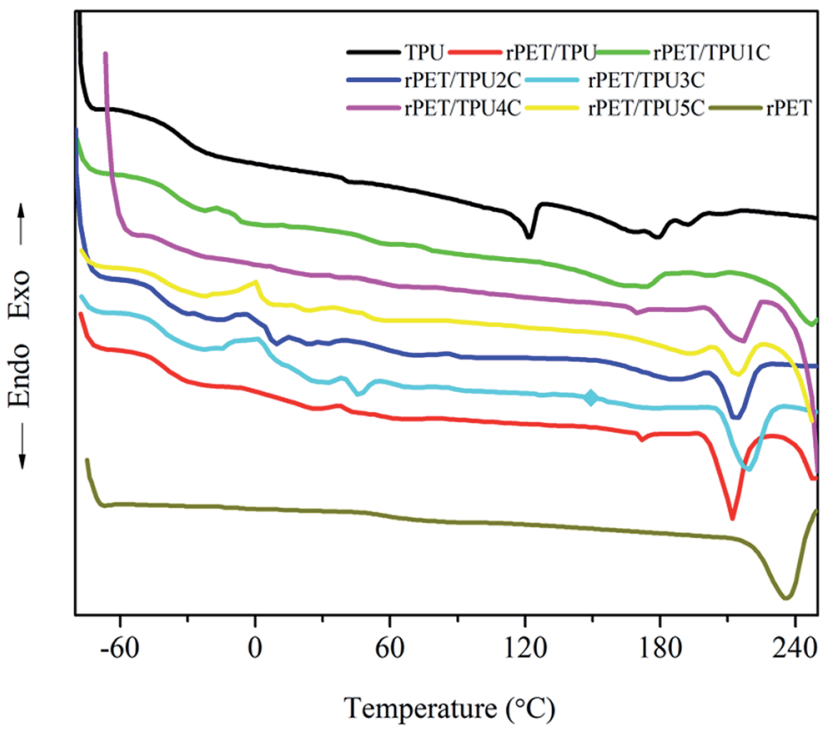

Fig. 4 DSC thermograms of fibers from pure $r P E T$, pure TPU and rPET/TPU and CNTs/rPET/TPU composites.

121.8 and $179.2{ }^{\circ} \mathrm{C}$. The $\mathrm{rPET} / \mathrm{TPU}$ and $\mathrm{CNTs} / \mathrm{rPET} / \mathrm{TPU}$ composites displayed only one glass transition each at $-29.6{ }^{\circ} \mathrm{C}$ and about $-34.5{ }^{\circ} \mathrm{C}$, respectively, indicating good compatibility between rPET and TPU materials in this work. The glass transition and crystallisation temperature of the TPU phase were affected by the presence of rPET. This was because the addition of rPET introduced a rigid benzene ring molecule, increasing the rigidity of the molecular chain, and resulting in an increase in the $T_{\mathrm{g}}$ of the composite. Although the previous study showed that CNTs can not alter the glass transition of TPU apparently, ${ }^{12}$ the change between the $T_{\mathrm{g}}$ of rPET/TPU and CNTs/ rPET/TPU composites clearly confirmed the mitigating effects of CNTs. This may be attributed to the offset effect of purified CNTs on the reaction of rPET, which decreased the rigidity of the molecular chain in the rPET/TPU composites. This could be due to the occurrence of the nucleation effect between the purified CNTs and the rPET matrix, which may increase the interfacial interaction between the CNTs and the rPET matrix and improve the rigidity of the rPET/TPU composite molecular chain. However, for the CNTs/rPET/TPU composites, with the

Table 2 A summary of the DSC cooling and heating curves for pure rPET, pure TPU and rPET/TPU and CNTs/rPET/TPU composites

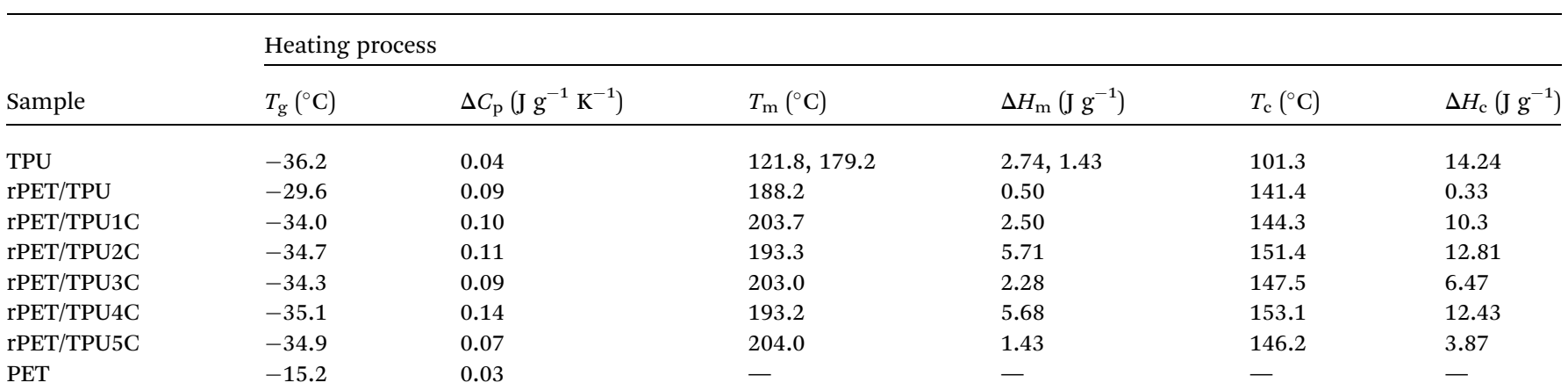


(a)

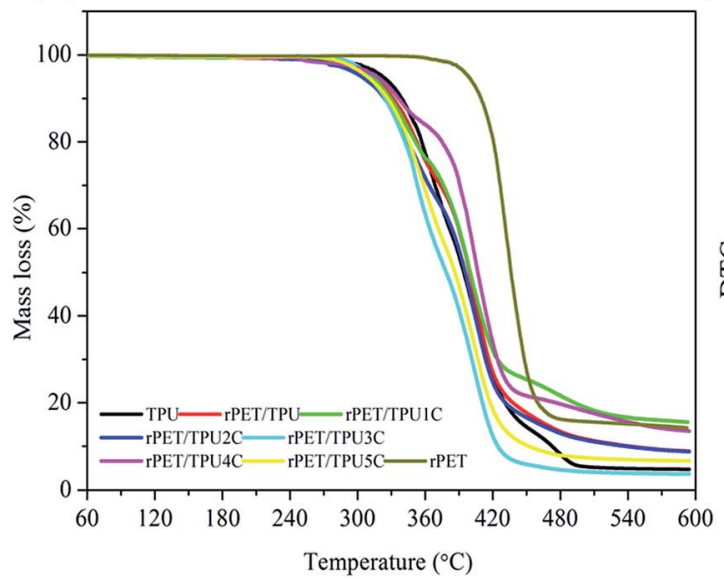

(b)

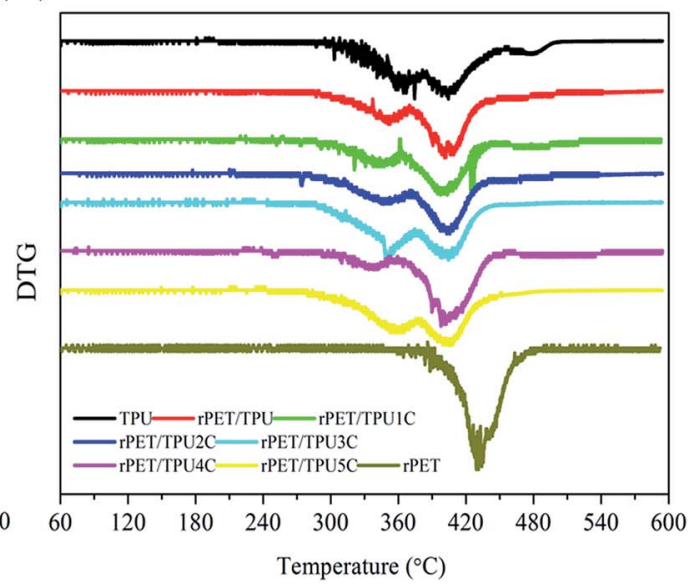

Fig. 5 (a) TGA and (b) DTG thermograms of pure rPET, pure TPU and rPET/TPU and CNTs/rPET/TPU composites.

addition of CNTs, the $T_{\mathrm{g}}$ of the composites showed no striking change. This may be because the amount of CNTs was too small to induce strong effects.

The heat capacity change $\left(\Delta C_{\mathrm{p}}\right)$ is proportional to the quantity of amorphous regions in the specimen..$^{30}$ With the addition of rPET, the $\Delta C_{\mathrm{p}}$ of rPET/TPU composites showed an increasing trend when compared to pure TPU. However, there was no striking change in the CNTs/rPET/TPU composites, which suggested that the introduction of rPET increased the amorphous region of the TPU matrix and the CNTs could not change the amorphous region of the rPET/TPU composites apparently. This result was consistent with the XRD analysis.

The thermal stability of the composites was investigated using TGA under a nitrogen atmosphere. The TGA and DTG curves of the prepared pure TPU, pure rPET and rPET/TPU and CNTs/rPET/TPU composites are shown in Fig. 5. The data, including the temperature for $5 \%\left(T_{5 \%}\right), 10 \%\left(T_{10 \%}\right)$ and $50 \%$ $\left(T_{50 \%}\right)$ mass loss and the maximum weight loss rate $\left(T_{\max }\right)$, are summarized in Table 3. As shown in Fig. 5, there were two main degradation stages from 30 to $600{ }^{\circ} \mathrm{C}$ in the pure TPU and composite samples. The first decomposition stage, between $300{ }^{\circ} \mathrm{C}$ and $375^{\circ} \mathrm{C}$, usually corresponds to the decomposition of urethane groups (-NH-COO-) in the hard segment. ${ }^{31}$ The second stage, between $380^{\circ} \mathrm{C}$ and $410^{\circ} \mathrm{C}$, may be ascribed to the polyol segments. ${ }^{27}$ Pure rPET displayed only one decomposition stage, between $380{ }^{\circ} \mathrm{C}$ and $480{ }^{\circ} \mathrm{C}$, corresponding to the decomposition of polyol segments. This result suggests that rPET and CNTs can not change the thermal degradation mechanism of polymer composites. From Table 3 and Fig. 5a, it can be seen that $T_{5 \%}$ and $T_{10 \%}$ for the rPET/TPU and CNTs/rPET/ TPU composites were lower than that for pure TPU and pure rPET, indicating that the thermal stability of TPU and rPET was better than that of all the composites. This may suggest that the existence of rPET affected the formation and degradation of urethane groups (-NH-COO-) in the hard segment of the TPU matrix, as seen in the XRD analysis. The change in the onset degradation temperature among the CNTs/rPET/TPU composites depended on the content and distribution status of the CNTs, a more even distribution resulted in better stability and interfacial adhesion in the composites, which can also be proved using SEM analysis. However, in the second decomposition, with the addition of CNTs, the thermal stability of the CNTs/rPET/TPU composites showed an increasing trend. When the CNT content exceeded $0.5 \mathrm{wt} \%$, the composite had a better thermal stability when compared to pure TPU. For example, when the weight loss was $50 \%$, the temperature of the rPET/ TPU2C sample was at $395.5{ }^{\circ} \mathrm{C}$ but for pure TPU it was at about $394.28^{\circ} \mathrm{C}$. This may be attributed to the $\mathrm{OH}$ group of the CNTs obtained by the acidizing treatment. The larger the amount of CNTs, the greater the formation of polyol segments.

\subsection{Dynamic mechanical properties}

In order to further study the morphology of the composites, the dynamic mechanical properties of the composites were investigated using DMA measurements. Fig. 6 shows the curves of the storage modulus $\left(E^{\prime}\right)$ and tan delta for pure TPU, pure rPET and rPET/TPU and CNTs/rPET/TPU composites versus temperature. The corresponding parameters are shown in Table 4.

Table 3 The main decomposition of pure TPU, pure rPET and rPET/TPU and CNTs/rPET/TPU composites

\begin{tabular}{|c|c|c|c|c|c|c|c|c|}
\hline Sample & TPU & rPET/TPU & rPET/TPU1C & rPET/TPU2C & rPET/TPU3C & rPET/TPU4C & rPET/TPU5C & rPET \\
\hline$T_{5 \%}\left({ }^{\circ} \mathrm{C}\right)$ & 322.10 & 310.15 & 308.76 & 307.82 & 312.19 & 312.73 & 317.47 & 398.72 \\
\hline$T_{10 \%}\left({ }^{\circ} \mathrm{C}\right)$ & 339.27 & 324.36 & 326.84 & 324.04 & 331.71 & 329.46 & 336.51 & 409.64 \\
\hline$T_{\max }\left({ }^{\circ} \mathrm{C}\right)$ & 404.78 & 403.20 & 403.73 & 403.74 & 401.98 & 400.40 & 403.71 & 429.4 \\
\hline
\end{tabular}


(a)

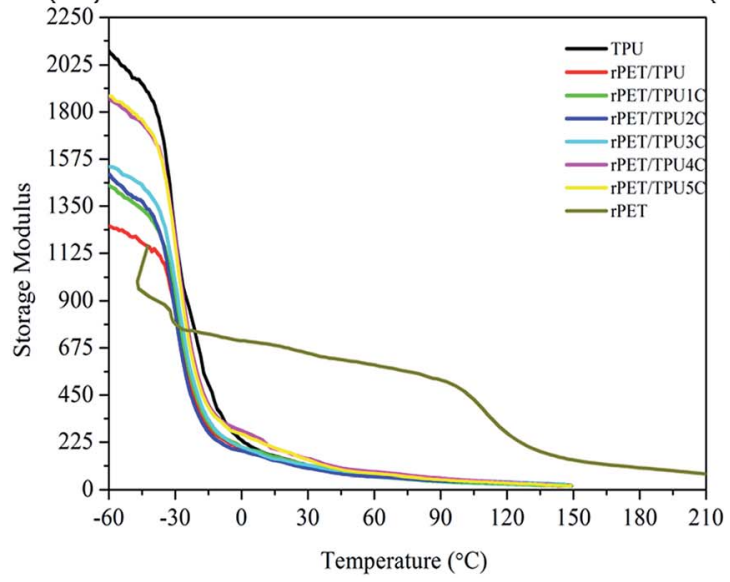

(b)

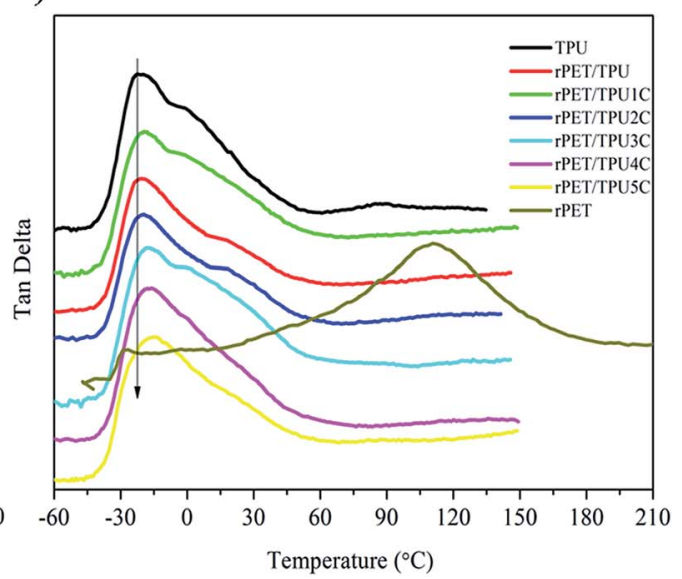

Fig. 6 Dynamic mechanical properties of pure rPET, pure TPU and rPET/TPU and CNTs/rPET/TPU composites. (a) Storage modulus, (b) tan delta.

Table 4 Glass transition parameters of pure TPU, pure rPET and rPET/TPU and CNTs/rPET/TPU composites obtained from peaks in the tan delta vs. temperature curves via DMA measurements

\begin{tabular}{lllllllll}
\hline Sample & TPU & rPET/TPU & rPET/TPU1C & rPET/TPU2C & rPET/TPU3C & rPET/TPU4C & rPET/TPU5C & rPET \\
\hline$T_{\mathrm{g}}\left({ }^{\circ} \mathrm{C}\right)$ & -22.40 & -15.60 & -19.51 & -19.53 & -17.75 & -16.10 & -20.12 & -26.62
\end{tabular}

From Fig. 6a, it can be seen that pure TPU displayed the highest storage modulus and the rPET/TPU composites showed a poor modulus, indicating that the existence of rPET resulted in poor mechanical properties. This was because, as shown in the TGA and XRD analysis, rPET affected the formation of urethane groups (-NH-COO-) in the hard segment of the TPU matrix and induced the appearance of a discontinuous TPU phase, which caused the poor mechanical properties. For the CNTs/rPET/TPU composites, the modulus showed an apparently increasing trend with an increase in CNT content, especially at temperatures below $T_{\mathrm{g}}$.

As shown in Fig. $6 \mathrm{~b}$ and Table 4, only one peak can be seen in all of the curves, suggesting good compatibility between rPET and TPU materials. The $T_{\mathrm{g}}$ of the rPET/TPU composites was higher than that of pure TPU and rPET, indicating that the introduction of rPET increased the molecular chain rigidity of the TPU matrix. This can possibly be attributed to the lower mobility of the soft segments in TPU confined in the rigid continuous PET phase. ${ }^{32}$ With the increase of CNTs, the $T_{\mathrm{g}}$ of CNTs/rPET/TPU composites showed a decreasing trend. This may be because the existence of purified CNTs allows interfacial adhesion between the CNTs and rPET, which offsets the rigid action from rPET to some extent, resulting in better flexibility of the polymer chain. The results are in agreement with the DSC analysis.

\subsection{Mechanical properties}

The mechanical properties of the composites are associated with composition and morphology (Table 5). ${ }^{6,32,33}$ As depicted in Fig. 7, all of the samples showed typical elastomeric behavior and no yield points, i.e. the relationship between stress and strain was directly proportional. For the CNTs/rPET/TPU composites, the tensile strength gradually increased with the increase in CNT content, which may be attributed to the excellent mechanical properties of CNTs. Table 5 shows an elongation of about $320 \%$ for pure TPU, an elongation of about $100 \%$ for the composites with $0.75,1$ and $2 \mathrm{wt} \%$ CNTs, and an elongation below $80 \%$ for the composites with 0 to $0.5 \mathrm{wt} \%$, indicating that the existence of rPET induced the appearance of a discontinuous TPU phase and the CNTs displayed an offset effect on the reaction of rPET. There was interfacial adhesion between PET and the purified CNTs, which may lead to the change in the mechanical properties, which can also be observed using DMA analysis. Higher interfacial adhesion may induce an increase in the tensile strength and elongation, and lower interfacial adhesion may induce a significant decline in

Table 5 The mechanical properties of pure TPU and rPET/TPU and CNTs/rPET/TPU composites

\begin{tabular}{|c|c|c|c|c|}
\hline Sample & $\begin{array}{l}\text { Tensile strength } \\
(\mathrm{MPa})\end{array}$ & $\begin{array}{l}\text { Elongation at } \\
\text { break }(\%)\end{array}$ & $\begin{array}{l}\text { Elastic } \\
\text { modulus } \\
(\mathrm{MPa})\end{array}$ & Shore A \\
\hline TPU & 176.12 & 313.08 & 8.43 & - \\
\hline rPET/TPU & 72.82 & 29.51 & 78.97 & 80.3 \\
\hline rPET/TPU1C & 83.73 & 70.91 & 45.86 & 83.1 \\
\hline rPET/TPU2C & 85.35 & 44.70 & 78.26 & 83.9 \\
\hline rPET/TPU3C & 90.87 & 106.53 & 90.28 & 84.3 \\
\hline rPET/TPU4C & 107.98 & 96.74 & 57.37 & 89.9 \\
\hline rPET/TPU5C & 137.65 & 116.89 & 81.18 & 93.1 \\
\hline
\end{tabular}




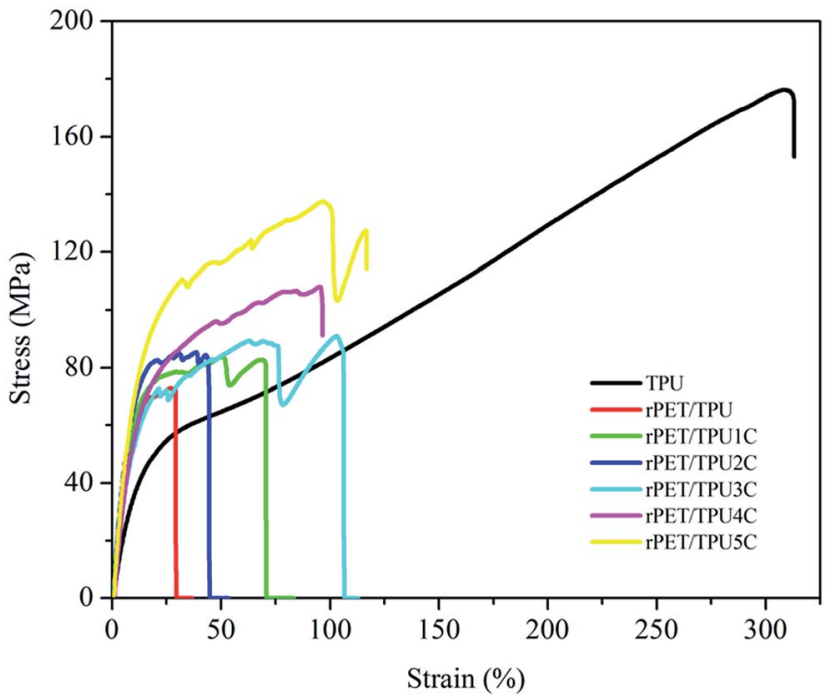

Fig. 7 Stress-strain curves for pure TPU and rPET/TPU and CNTs/ rPET/TPU composites.

elongation. The elastic modulus of the CNTs/rPET/TPU composites with a CNT content ranging from 0 to $2 \mathrm{wt} \%$ was more than 6 times higher than that of pure TPU, and similar results also appear in other polymer nanocomposite systems, such as PET/LDPE blends..$^{33}$ From Table 5, it can be seen that with increasing CNT content, the hardness of the composites has a similar tendency to the tensile strength because the introduction of CNTs contributes to interfacial adhesion in the matrix and improves the hardness of the composites. In this section, the results show that one can modify the material properties via blending modification to obtain satisfactory properties of hardness and good tensile strength under the right proportion conditions.

\section{Conclusions}

In this work, thermoplastic polyurethane (TPU) was blended with recycled polyethylene terephthalate (rPET) to prepare $\mathrm{rPET} /$ thermoplastic polyurethane (TPU) composites. CNTs/rPET/TPU composites with different CNT amounts were prepared. The results showed that TPU was compatible with rPET. The existence of rPET changed the crystalline phase and affected the glass transition and crystallisation temperature of the TPU matrix. The rPET/TPU composites displayed poor thermal stability and tensile properties when compared to pure TPU. The addition of CNTs had no effect on the crystalline phase of rPET/ TPU composites. This was because of the occurrence of interfacial adhesion between CNTs and rPET, the CNTs displayed an offset effect on the reaction of rPET and decreased the rigidity of the molecular chain in the rPET/TPU composites. The thermal stability and tensile strength of the CNTs/rPET/TPU composites improved with an increase in CNT content.

\section{Conflicts of interest}

There are no conflicts to declare.

\section{Acknowledgements}

The authors acknowledge the financial support provided by Programs for New Century Excellent Talents in University of Ministry of Education of China (Grant No. NCET-12-1045), Shaanxi Programs for Science and Technology Development (Fund No. 2010K01-096), Key Program for Innovation Team in Shaanxi Province, Ph.D. Innovation fund projects of Xi'an University of Technology (Fund No. 310-252071501), and Program for Innovation Team in Xi'an University of Technology (Grant No. 108-25605T401).

\section{References}

$1 \mathrm{H}$. Chen, Z. Liu and P. Cebe, Chain confinement in electrospun nanofibers of PET with carbon nanotubes, Polymer, 2009, 50, 872-880.

2 Y. Asakuma, K. Nakagawa and K. Maeda, Theoretical study of the transesterification reaction of polyethylene terephthalate under basic conditions, Polym. Degrad. Stab., 2009, 94, 240245.

3 G. M. M. Sadeghi and M. Sayaf, From PET waste to novel polyurethanes, Mater. Recyc. Trend. Persp., 2012.

4 I. M. Nikbin, R. R. Saman and H. Allahyari, Feasibility study of waste poly ethylene terephthalate (PET) particles as aggregate replacement for acid erosion of sustainable structural normal and lightweight concrete, J. Cleaner Prod., 2016, 126, 108-117.

5 S. R. Shukla, A. M. Harad and L. S. Jawale, Chemical recycling of PET waste into hydrophobic textile dyestuffs, Polym. Degrad. Stab., 2009, 94, 604-609.

6 C. Jiang, X. Xu and Z. Li, Application of in situ microfibrillization to recycling ultraviolet-aged poly(ethylene terephthalate) (PET) and high density polyethylene (HDPE), J. Macromol. Sci., Part B: Phys., 2007, 1, 10-25.

7 S. Park, K. Park and K. Suh, Compatibilizing effect of isocyanate functional group on polyethylene terephthalate/ low density polyethylene blends, J. Polym. Sci., Part B: Polym. Phys., 2015, 36, 447-453.

8 K. Jayanarayanan, S. Thomas and K. Joseph, Effect of blend ratio on the mechanical and sorption behaviour of polymer-polymer microfibrillar composites from lowdensity polyethylene and polyethylene terephthalate, $J$. Reinf. Plast. Compos., 2012, 31, 549-562.

$9 \mathrm{X}$. Yi and L. Xu, Morphology and properties of isotactic polypropylene/poly(ethylene terephthalate) in situ microfibrillar reinforced blends: influence of viscosity ratio, Eur. Polym. J., 2010, 46, 719-730.

10 C. Ji, M. Xie and B. Chang, $\beta$-Crystal in injection moulded poly(ethylene terephthalate) fibre/isotactic polypropylene composite, Composites, Part A, 2013, 46, 26-33.

11 R. Borg, O. Baldacchino and L. Ferrara, Early age performance and mechanical characteristics of recycled PET fibre reinforced concrete, Constr. Build. Mater., 2016, 108, 29-47. 
12 J. Chen, Z. X. Zhang and W. B. Huang, Carbon nanotube network structure induced strain sensitivity and shape memory behavior changes of thermoplastic polyurethane, Mater. Des., 2015, 69, 105-113.

13 V. Jašo, M. V. Rodić and Z. S. Petrović, Biocompatible fibers from thermoplastic polyurethane reinforced with polylactic acid microfibers, Eur. Polym. J., 2015, 63, 20-28.

14 R. Manfred, P. Jurgen and R. Thomas, Reactive polymer blends with thermoplastic polyurethane, Makromol. Chem., Macromol. Symp., 1991, 50, 203-214.

$15 \mathrm{~S} . \mathrm{Xu}, \mathrm{W}$. Yu and M. Jing, Largely Enhanced Stretching Sensitivity of Polyurethane/CNTs Nanocomposites via Incorporation of Cellulose Nanofiber, J. Phys. Chem. C, 2017, 121, 4.

16 S. S. Mahapatra, S. K. Yadav and H. J. Yoo, Tailored and strong electro-responsive shape memory actuation in carbon nanotube-reinforced hyperbranched polyurethane composites, Sens. Actuators, B, 2014, 193, 384-390.

17 D. Alhazov, A. Gradys and P. Sajkiewicz, Thermo-mechanical behavior of electrospun thermoplastic polyurethane nanofibers, Eur. Polym. J., 2013, 49, 3851-3856.

18 J. Chen, Z. X. Zhang and W. B. Huang, Carbon nanotube network structure induced strain sensitivity and shape memory behavior changes of thermoplastic polyurethane, Mater. Des., 2015, 69, 105-113.

19 J. Z. Xu, G. J. Zhong and B. S. Hsiao, Low-dimensional carbonaceous nanofiller induced polymer crystallization, Prog. Polym. Sci., 2014, 39, 555-593.

20 B. W. Steinert and D. R. Dean, Magnetic field alignment and electrical properties of solution cast PET-carbon nanotube composite films, Polymer, 2009, 50, 898-904.

21 S. Mallakpour and V. Behranvand, Application of recycled PET/carboxylated multi-walled carbon nanotube composites for $\mathrm{Cd}^{2+}$, adsorption from aqueous solution: a study of morphology, thermal stability, and electrical conductivity, Colloid Polym. Sci., 2017, 295, 1-10.

22 R. Cardinaud and T. Mcnally, Localization of MWCNTs in PET/LDPE blends, Eur. Polym. J., 2013, 49, 1287-1297.
23 Y. Shi, W. Zhang and J. Yang, Super toughening of the poly(Llactide)/thermoplastic polyurethane blends by carbon nanotubes, RSC Adv., 2013, 3, 26271-26282.

24 Z. Liu, H. Bai and Y. Luo, Achieving a low electrical percolation threshold and superior mechanical performance in poly(L-lactide)/thermoplastic polyurethane/ carbon nanotubes composites via tailoring phase morphology with the aid of stereocomplex crystallites, $R S C$ Adv., 2017, 7, 11076-11084.

$25 \mathrm{X}$. Zhou, C. Fang and Y. Li, Preparation and characterization of $\mathrm{Fe}_{3} \mathrm{O}_{4}$-CNTs magnetic nanocomposites for potential application in functional magnetic printing ink, Composites, Part B, 2016, 89, 295-302.

26 V. García-Pacios and V. Costa, Affect of polydispersity on the properties of waterborne polyurethane dispersions based on polycarbonate polyol, Int. J. Adhes. Adhes., 2010, 30, 456-465.

27 C. Q. Fang, W. Q. Lei and X. Zhou, Preparation and characterization of waterborne polyurethane containing PET waste/PPG as soft segment, J. Appl. Polym. Sci., 2015, 132, 45.

28 M. Endo and K. Takeuchi, Stacking nature of grapheme layers in carbon nanotubes and nanofibres, J. Phys. Chem. Solids, 1997, 58, 1707.

29 S. Hashmi, H. Prasad and R. Abishera, Improved recovery stress in multi-walled-carbon-nanotubes reinforced polyurethane, Mater. Des., 2015, 67, 492-500.

30 X. Zhou, C. Q. Fang and W. Q. Lei, Thermal and Crystalline Properties of Waterborne Polyurethane by in situ water reaction process and the potential application as biomaterial, Prog. Org. Coat., 2017, 1-10.

31 X. Lai, Y. Shen and L. Wang, Preparation and properties of self-crosslinkable polyurethane/silane hybrid emulsion, $J$. Polym. Res., 2011, 18, 2425-2433.

32 V. Jašo, M. Cvetinov and S. Rakić, Bio-plastics and elastomers from polylactic acid/thermoplastic polyurethane blends, J. Appl. Polym. Sci., 2014, 131, 547-557.

33 S. Fakirov, H. Kamo and M. Evstatiev, Microfibrillar reinforced composites from PET/LDPE blends: morphology and mechanical properties, J. Macromol. Sci., Part B: Phys., 2004, 43, 775-789. 